\title{
Heavy quark limit in the model with confined light quarks and infrared heavy quark propagators
}

\author{
M. A. Ivanov* and T. Mizutani \\ Department of Physics \\ Virginia Polytechnic Institute and State University \\ Blacksburg, VA 24061
}

November 21, 2018

\begin{abstract}
We have studied the weak decay constants and the Isgur-Wise form factor of the $\mathrm{B}$ and $\mathrm{D}$ mesons in the heavy quark limit, by employing a relativistic quark confinement model. It is an attempt to improve our previous work within the same line of thinking, but by incorporating a couple of novel aspects. First, the infrared behavior of the heavy quark is considered by modifying its conventional propagator in terms of a single parameter $\nu$. Second, the mass difference of the heavy meson and heavy quark: $E=m_{H}-M_{Q}$ has been included. It is found that the weak decay constants depend strongly on the mass difference $\mathrm{E}$ with a relatively mild $\nu$ dependence. As for the Isgur-Wise function it is controlled more sensitively by the infrared parameter $\nu$, leading to its suppression at maximum meson recoil.
\end{abstract}

PACS codes: 13.20, 13.28, 14.48.N

${ }^{*}$ Permanent address: Laboratory of Theoretical Physics, Joint Institute for Nuclear Research, Head Post Office P.O.Box 79, 101000 Moscow, Russia 


\section{Introduction}

The theoretical treatment of heavy-quark hadrons: those containing a heavy ( $b$ or $c$ ) quark $\left(M_{Q}>>\Lambda_{Q C D}\right)$, is considerably simplified due to a new heavy quark (spin-flavor) symmetry [1]. Under this symmetry a heavy-quark meson may be pictured as a system of a cloud of light degrees of freedom (light quarks and gluons) surrounding the almost static color source (a heavy quark) whose four-momentum being quite close to its onmass-shell value. In practice, it allows for expressing all the $B \rightarrow D\left(D^{*}\right) l \nu$ decay form factors in terms of a single universal form factor: the Isgur-Wise function (IWF), which is determined primarily by the dynamics of the light degrees of freedom.

The systematical study of this symmetry is provided by the heavy-quark effective theory (HQET) developed by Georgi et al. [2]. In HQET the heavy degrees of freedom are integrated out, while their effects as virtual particles are taken into account by introducing non-renormalizable higher-dimension operators proportional to the inverse powers of the heavy quark mass [3]. However, IWF cannot be directly obtained within this scheme since it is essentially determined by the physics in the non-perturbative domain. Thus, to work this quantity out various quark models [4], [6], [7], [8], [9], [10], [11], QCD sum rules [12], [13], [14], [15], and lattice calculations [16], [17] have been employed so far. The present work is devoted to the extension of our previous calculation of IWF within a relativistic confined quark model [7]. To constrain the model parameters we will also calculate the weak decay constants of the $B$ and $D$ mesons. In order to set a basic stage for our study, we shall first briefly review some of the model results to date in the following.

In the nonreltivistic confined quark model [⿴囗十 IWF is expressed in terms of the overlap integral of the initial and final meson wave functions of the Gaussian form which are determined by fit to meson spectra (note that heavy quark mass is assumed to be infinity here). With $w \equiv v \cdot v^{\prime}$ where $v\left(v^{\prime}\right)$ is the four-velocity of the initial (final) heavy-quark meson, it reads

$$
\xi(w)=\exp \left\{-\rho^{2}(w-1)\right\}
$$

The slope parameter obtained from the meson spectroscopy is $\rho^{2}=-\xi^{\prime}(1)=0.33$. Anticipating that the result would be insufficient for large recoil, the authors of [1] have 
introduced a universal reduction factor (from a fit to the pion electromagnetic form factor) which has resulted in an increase in the slope parameter: $\rho^{2} \approx 0.64$. We note here a recent claim [5] that a due consideration of the relativistic recoil correction and the Wigner rotation of the spin of the light quark should increase the slope parameter to greater than unity.

To improve certain aspects of nonrelativistic approachs a simple relativistic-oscillator model with the light-front variables was adopted to find IWF [6],

$$
\xi(w)=\frac{2}{w+1} \exp \left\{-\left(2 \rho^{2}-1\right) \frac{w-1}{w+1}\right\} .
$$

Here, the slope parameter is related to the transverse momentum distribution of the light quark inside the heavy meson. By comparing with the data its value has been extracted as $\rho \sim 1.14 \pm 0.23$.

We next discuss other quark model approaches with or without due consideration to confinement. First, a simple model based on quark loop graphs with an ad hoc form factor at each heavy-meson heavy-quark light-antiquark vertex was proposed in Ref. [8]. It was found that the physical quantities (including IWF) are quite sensitive to the mass difference; $E \equiv m_{H}-M_{Q}$, of the heavy meson and heavy quark when it exceeds $m_{q}$ : the light-quark (constituent) mass of the model. Then the mass function (self energy), decay form factor (IWF), etc. of the heavy meson made up of a heavy-quark light-antiquark loop develop an imaginary part which becomes of the same order of magnitude as the real part. This is of course due to the absence of the quark confinement in the model. Eventually the imaginary part has been discarded completely by arguing that its neglect would mimic the quark confinement. To date, the justification of such a procedure is not known.

A modified MIT bag model was applied to the calculation of IWF for the ground state to ground state semi-leptonic decays involving the $b \rightarrow c$ transition [10]. The evaluation of the overlap integral yielded a result, which is claimed to be well approximated by the following form 13

$$
\xi(w)=\left[\frac{2}{w+1}\right]^{2+\frac{0.6}{w}}
$$


The heavy-quark meson wave function was obtained by solving the bound state BetheSalpeter equation with a modified one-gluon-exchange ladder approximation [11]. The decay constant $f_{B}$ and IWF were then calculated from the wave function. The slope parameter at zero recoil was found to be rather large: $\rho^{2}=1.8-2.0$, whereas the weak decay constant turned out very small, $f_{B} \approx 50 \mathrm{MeV}$. Presumably, the one-gluon-exchange may not be very realistic for generation of the heavy meson bound state (this interaction alone does not confine the quarks).

Our previous approach for IWF [7] was based upon the quark confinement model (QCM) which incorporate the confinement of light quarks by devising a quark propagator that has no singularities, thus forbidding the production of a free quark [25]. This model then allows one to perform covariant calculations of Feynman diagrams with dressed light quark propagators free of simple pole. The calculation has given, in the heavy quark limit: $M_{Q}=m_{H} \rightarrow \infty$, the IWF to decrease slower than that obtained by many other approaches except the bounds [19] to be discussed below,

$$
\xi(w)=\frac{1}{1+R}\left[\frac{2}{1+w} R+\frac{1}{\sqrt{w^{2}-1}} \ln \left[w+\sqrt{w^{2}-1}\right]\right],
$$

with the parameter fixed by the light quark confinement function (see the next section) to be $R=0.65$. The corresponding slope parameter was extracted to be equal to $\rho^{2}=0.43$, quite small as compared with the valued obtained from a fit to the data by the relativistic oscillater model as discussed above. It should be mentioned that the IWF was obtained also with finite heavy quark masses, and the result turned out to be very close to the one in the heavy quark limit.

It should be useful at this point to refer to a rather peculiar model [9] which, in part, has motivated our present investigation. It is based upon the observation of the possible infrared behavior of the heavy quark propagator. The physics here is not quite dynamical but mostly geometrical, i.e. in terms infraparticle (heavy quark) propagation only without taking into account the light degrees of freedom. The obtained IWF has the form

$$
\xi(w)=1-\exp \left\{-\frac{1.02}{\sqrt{w^{2}-1}}\right\}
$$

with the slope at zero recoil equal to zero. The model does not appear particularly 
realistic, but its consideration of the infrared propagator is quite novel which we have adopted in our present work.

Different from various quark model approaches discused so far, the QCD (spectral) sum rule [18] appears to be the only alternative so far in effectively dealing the long distance physics of the heavy mesons, aside from the direct numerical simulation by lattice QCD. This approach is based upon the hypothesis of quark-hadron duality in the calculation of physical correlators and is combined with HQET to simplify the physics. After several simplifying assumptions the first sum rule calculation [12] found

$$
\xi(w) \approx \exp \left\{-0.37 \sqrt{w^{2}-1}\right\}
$$

with the infinite slope parameter.

Later, this approach was improved [13] [14] [15], by including the next to leading order renormalization group improvement, finite heavy-quark mass effects, etc. It was found that these effects are quite large for the weak decay constant and makes the Dmeson as much as about 40\%, 13 causing a large deviation from the asymptotic scaling law $f_{D} \sqrt{m_{D}}=f_{B} \sqrt{m_{B}}$. Also a rather strong dependence of this quantity on the mass difference $E=m_{H}-M_{Q}$ was observed. As for IWF the E dependence was fairly weak or cancel out completely. The main uncertainty brought in the calculation of this quantity comes from the QCD perturbative estimate of the higher-resonance contributions to the spectral function in the dispersion integral representation of the hadronic correlator. It turned out that the different choice of the spectral function and of its integration domain produces the values of the IWF form factor at maximum recoil varing in the region $0.40 \leq \xi\left(w_{\max }\right) \leq 0.75$. It was also shown that the infinite slope parameter of [12] has resulted from a naïve choice of the integration domain of the spectral function. There appears, however, no single best choice for this domain. The "best" result compared with the experimental data [37] may be parametrized as in eq.(3), giving $\rho^{2}=1.3$.

The lattice QCD has been exploited by two groups [16] and [17 in the study of heavy meson physics. The IWF was obtained by calculating the elastic D amplitude (or form factor $<D^{\prime}\left|\bar{c} \gamma_{\mu} c\right| D>$, in the quenched approximation. Fitting the result to the relativistic-oscillator parametrization Eq.(2) gives 


$$
\rho^{2}= \begin{cases}1.41 \pm 0.19 \pm 0.41 & \text { [16] } \\ 1.2_{-3}^{+7} & \text { [17] }\end{cases}
$$

Lastly, we should mention that the bounds on the IWF were studied without relying on particular models, but only with the consideration of analyticity and dispersion relation for the heavy meson elastic form factor assuming the heavy quark symmetry and the hardon-quark duality [19]. It was claimed that the bounds is very strongly constrained. These bounds prohibit the IWF from falling by less than $12 \%$ and more than $21 \%$ from zero to maximum recoil. The lower bound conflicts with many model predictions. It has been argued by [20, 21], 22], and [23] that the careful inclusion of heavy quark-antiquark bound states, lying below the heavy meson pair production threshold, may gives no such small lower bound. The authors of [19 recently included the effect of the $\Upsilon$ states below the $B \bar{B}$ threshold [24], claiming that the rigorous upper bound for the IWF slope at zero recoil is now $\rho^{2} \approx 6.0$, although, on phenomenological grounds, it may be diminished to $\rho^{2} \leq 1.7$.

The main goal of the present paper is to improve our previous work [7] as we believe that our QCM approach possesses a sound dynamical background regarding its modelling of confined light quarks, successful in many static and non-static properties of the light hadrons 25]. Thus the present investigation will focus on how one should treat the behavior of the heavy quark within the context of this QCM.

\section{Quark propagators}

The quark confinement model (QCM) 25] is specified by the interaction Lagrangian inferred from the partial hadronization of the DCQ action. Then the transition between heavy meson $H$ and heavy $Q$ and light $q$ quarks is described by

$$
\mathcal{L}_{H}(x)=g_{H} H(x) \bar{Q}(x) \Gamma_{H} q(x)
$$

with the coupling constants $g_{H}$ defined by what is usually called the compositeness condition which means that the renormalization constant of the meson field is equal to zero: 


$$
Z_{H}=1-g_{H}^{2} \Pi_{H}^{\prime}\left(m_{H}^{2}\right)=0 .
$$

Here $\Pi_{H}^{\prime}$ is the derivative of the meson mass operator. The compositeness condition also provides the right normalization of the charge form factor $F_{H}(0)=1$. This could be readily seen from the Ward identity

$$
g_{H}^{2} \Pi_{H}^{\prime}\left(p^{2}\right)=g_{H}^{2} \frac{1}{2 p^{2}} p^{\mu} \frac{\partial \Pi_{H}\left(p^{2}\right)}{\partial p^{\mu}}=g_{H}^{2} \frac{1}{2 p^{2}} p^{\mu} T_{H H}^{\mu}(p, p)=F_{H}(0)=1 .
$$

with the three-point function $T^{\mu}\left(p, p^{\prime}\right)$ defined as

$$
T_{H H}^{\mu}\left(p, p^{\prime}\right)=\frac{3}{4 \pi^{2}} \int \frac{d^{4} k}{4 \pi^{2} i} \operatorname{tr}\left\{\Gamma S_{Q}\left(\not k-\not p^{\prime}\right) \gamma^{\mu} S_{Q}(\not k-\not p) \Gamma S_{q}(\not k)\right\} .
$$

The matrix elements describing the leptonic decays $H\left(H^{*}\right) \rightarrow e \nu(H=B, D)$, and semileptonic ones $B \rightarrow D\left(D^{*}\right) e \nu$ are written down as

$$
M^{\mu}\left\{H\left(H^{*}\right) \rightarrow e \nu\right\}=g_{H} T_{H}^{\mu}(p),
$$

where

$$
T_{H}^{\mu}(p)=\frac{3}{4 \pi^{2}} \int \frac{d^{4} k}{4 \pi^{2} i} \cdot \operatorname{tr}\left\{O^{\mu} S_{Q}(\not k-\not p) \Gamma S_{q}(\not k)\right\}
$$

and

$$
M_{B \rightarrow D\left(D^{*}\right) e \nu}^{\mu}=g_{B} g_{D\left(D^{*}\right)} T_{H H^{\prime}}^{\mu}\left(p, p^{\prime}\right)
$$

where

$$
T_{H H^{\prime}}^{\mu}\left(p, p^{\prime}\right)=\frac{3}{4 \pi^{2}} \int \frac{d^{4} k}{4 \pi^{2} i} \operatorname{tr}\left\{\gamma^{5} S_{Q^{\prime}}\left(\not k-\not p^{\prime}\right) O^{\mu} S_{Q}(\not k-\not p) \Gamma S_{q}(\not k)\right\} .
$$

Now we discuss the forms of quark propagators.

\section{(1) Confined light-quark propagator.}


Here we briefly explain the salient feature of QCM concerning the light-quark propagator. The principal assumption [25] is that the propagator is an entire analytical function in the complex-" $\not p$ " plane with the constituent mass $m_{q} \equiv z \Lambda$ being smeared by complex measure $d \sigma_{z}$ such that

$$
\int \frac{d \sigma_{z}}{\Lambda z-\not p}=\frac{1}{\Lambda}\left[a\left(-\frac{p^{2}}{\Lambda^{2}}\right)+\frac{\not p}{\Lambda} b\left(-\frac{p^{2}}{\Lambda^{2}}\right)\right]
$$

with the confinement functions $a(u)$ and $b(u)$ defined by

$$
a(u)=\int d \sigma_{z} \frac{z}{z^{2}+u^{2}} \quad b(u)=\int d \sigma_{z} \frac{1}{z^{2}+u^{2}} .
$$

The scale parameter $\Lambda$ characterizes the size of the confinement region. It has turned out 25] that the low-energy physics depends only on those quantities which involve the integrals of $a(u)$ and $b(u)$ together with $u^{\alpha} \quad(\alpha \geq 0)$ but not on the detailed shape of these functions. We have devized a simple choice of the confinement functions [25]:

$$
a(u)=a_{0} \exp \left(-u^{2}-a_{1} u\right) \quad b(u)=b_{0} \exp \left(-u^{2}+b_{1} u\right) .
$$

The parameters $a_{i}, b_{i}$, and $\Lambda$ have been determined from the best model description of hadronic properties at low energies and the following values were found:

$$
\begin{aligned}
& a_{0}=b_{0}=2, \quad a_{1}=1, \quad b_{1}=0.4, \\
& \text { and } \quad \Lambda=460 \mathrm{MeV},
\end{aligned}
$$

which describe various physical observables quite well [25].

\section{(2) Infrared heavy-quark propagator.}

Since a heavy quark (with mass $M_{Q}$ ) in a heavy meson is under the influence of soft gluons (which sets the scale $\Lambda_{Q C D}<<M_{Q}$ ), it may be regarded as nearly on its mass-shell where the infrared regime should take place for its propagation. The infrared behavior for one-fermion Green's function (propagator) has been investigated in various papers (see, 
for instance, [9], 26], and the references therein). The result is well-known only for abelian gauge theories:

$$
S(p) \sim(m-\not p-i \epsilon)^{-1-\nu}
$$

where $\nu=\left(\alpha_{S} / 4 \pi\right)(3-\lambda)$ with $\lambda$ being the gauge parameter. It seems quite resonable to see how sensitive the physical observables may be to this modification even though the integrals in Eqs.(10), (12), (14) have no infrared divergences. To make our present study simple, we choose the heavy quark propagator as in Eq.(19),

$$
S_{Q}(k+p)=\left(M_{Q}-\not k-\not p\right)^{-1-\nu}
$$

assuming that $\nu$ is a free parameter. Using the conventional notation for the heavy meson four-momentum $p=\left(M_{Q}+E\right) v$, with $v$ being the four-velocity of the heavy meson (or of the heavy quark since there is a velocity selection rule: [2], one readily finds that this propagator satisfies the form necessary for the correct heavy quark limit:

$$
S_{Q}(k+p)=\left[\frac{1}{M_{Q}-\not k-\not p}\right]^{1+\nu}=\frac{1}{2} \frac{1+\not p}{(-E-k v)^{1+\nu}}+O\left(\frac{1}{M_{Q}}\right) .
$$

The physical observables are defined by two parameters: the heavy meson heavy quark mass difference $E=m_{H}-M_{Q}$ which typifies the binding of the light and heavy quarks,

and $\nu$ characterizing the infrared behavior of the heavy quark as disscussed above. It should be mentioned here that in our previous work [7] we have not considered the effect of the non-vanishing $E$. In the heavy quark limit both the meson mass $m_{H}$ and heavy quark mass $M_{Q}$ are sent to infinity, so it does not appear to make sense to keep this value finite. However, it characterizes the binding of the light and heavy quarks, thus an very important parameter particularly in constraining the values of the weak decay constant, as we shall see later. We also note that this quantity plays a significant role in other approaches.

\section{Weak decay constants and IW form factor}

The calculation of the two- and three-point functions (see Eqs.(10),(12), and (14)) is considerably simplified by taking only the leading order in the $1 / M_{Q}$ expansion (the 
heavy quark limit) since the calculations of the traces and the integrations over internal momentum $k$ factorize in this limit due to the following identities:

$$
\begin{aligned}
& \operatorname{tr}\left[O^{\mu}(1+\not \varnothing) \Gamma(z+\not k)\right]=\operatorname{tr}\left[O^{\mu}(1+\not b) \Gamma(z+\not b k v)\right]=\operatorname{tr}\left[O^{\mu}(1+\not \varnothing) \Gamma\right](z-k v) \\
& \operatorname{tr}\left[\Gamma(1+\not b) \gamma^{\mu}(1+\not b) \Gamma(z+\not k)\right]=2 v^{\mu} \operatorname{tr}[\Gamma \Gamma](z-k v) \\
& \operatorname{tr}\left[\Gamma\left(1+\not \not^{\prime}\right) O^{\mu}(1+\not x) \gamma^{5}(z+\not k)\right] \\
& =\operatorname{tr}\left[\Gamma\left(1+\not \not^{\prime}\right) O^{\mu}(1+\not)^{5} \gamma^{5}\left(z+\frac{k v+k v^{\prime}}{2(w+1)}\left(\not \varnothing+\not \not^{\prime}\right)-\frac{k v-k v^{\prime}}{2(w-1)}\left(\not \varnothing-\not \not^{\prime}\right)\right)\right] \\
& =\operatorname{tr}\left[\Gamma\left(1+\not \not^{\prime}\right) O^{\mu}(1+\not) \gamma^{5}\right]\left(z-\frac{k v+k v^{\prime}}{(w+1)}\right)
\end{aligned}
$$

where $w=v \cdot v^{\prime}$. Here we have taken into account that $\{\Gamma, \not p\}=0$ for both $\Gamma=\gamma^{5}$ and $\gamma^{\nu}$, which follows from the transversality of the vector field.

Using these identities in the Eqs.(10), (12) and (14) gives

$$
\begin{aligned}
T_{H H}^{\mu}(p, p) & =2 p^{\mu}\left(\frac{\Lambda}{m_{H}}\right)\left(\frac{1}{2 \Lambda^{\nu}}\right)^{2} \frac{3}{4 \pi^{2}} J_{3}^{(\nu)}\left(\frac{E}{\Lambda}, 1\right) \cdot L_{0}, \\
T_{H}^{\mu}(p) & =\Lambda\left(\frac{\Lambda}{m_{H}}\right) \frac{1}{2 \Lambda^{\nu}} \frac{3}{4 \pi^{2}} J_{2}^{(\nu)}\left(\frac{E}{\Lambda}\right) \cdot L_{1}^{\mu}, \\
T_{H H^{\prime}}^{\mu}\left(p, p^{\prime}\right) & =\frac{\Lambda}{\left(2 \Lambda^{\nu}\right)^{2}} \frac{3}{4 \pi^{2}} J_{3}^{(\nu)}\left(\frac{E}{\Lambda}, w\right) \cdot L_{2}^{\mu} .
\end{aligned}
$$

Here we use notation:

$$
\begin{gathered}
L_{0}= \begin{cases}I & \left(\Gamma=\gamma^{5}\right) \\
g^{\alpha \beta} & \left(\Gamma=\gamma^{\alpha}\right)\end{cases} \\
L_{1}^{\mu}= \begin{cases}p^{\mu} & \left(\Gamma=\gamma^{5}\right) \\
M \epsilon^{\mu} & \left(\Gamma=\gamma^{\alpha}\right)\end{cases}
\end{gathered}
$$




$$
L_{2}^{\mu}= \begin{cases}\left(v+v^{\prime}\right)^{\mu} & \left(\Gamma=\gamma^{5}\right) \\ i \varepsilon^{\mu \epsilon v^{\prime} v}+v^{\prime \mu}(v \epsilon)-\epsilon^{\mu}(w+1) & \left(\Gamma=\gamma^{\alpha}\right)\end{cases}
$$

where $\epsilon$ is the polarization vector. The structural integrals $J_{2}^{(\nu)}$ and $J_{2}^{(\nu)}$ are defined as

$$
\begin{aligned}
J_{2}^{(\nu)}(E) & =\int \frac{d^{4} k}{\pi^{2} i} \int d \sigma_{z} \frac{z-k v}{\left(z^{2}-k^{2}\right)(-E-k v)^{1+\nu}} \\
J_{3}^{(\nu)}(E, w) & =\int \frac{d^{4} k}{\pi^{2} i} \int d \sigma_{z} \frac{z-\left(k v+k v^{\prime}\right) /(w+1)}{\left(z^{2}-k^{2}\right)(-E-k v)^{1+\nu}\left(-E-k v^{\prime}\right)^{1+\nu}}
\end{aligned}
$$

and they are calculated in the Appendix.

The $H Q \bar{q}$ coupling constant is determined from Eq.(8) and may be written down as

$$
g\left(m_{H}\right)=2 \Lambda^{\nu} \frac{4 \pi}{\sqrt{3}} \sqrt{\frac{m_{H}}{\Lambda}} \sqrt{\frac{1}{J_{3}^{(\nu)}(E / \Lambda, 1)}} .
$$

Finally, the matrix elements of the leptonic and semileptonic decays are written as

$$
\begin{aligned}
M_{H\left(H^{*}\right) \rightarrow e \nu}^{\mu} & =f_{H} \cdot L_{1}^{\mu} \\
M_{B \rightarrow D\left(D^{*}\right) e \nu}^{\mu} & =\sqrt{m_{B} m_{D}} \xi(w) \cdot L_{2}^{\mu}
\end{aligned}
$$

with the weak decay constant $f_{H}$ and the IWF given by

$$
\begin{aligned}
f_{H} \sqrt{m_{H}} & =\Lambda^{3 / 2} \frac{\sqrt{3}}{2 \pi} \frac{J_{2}^{(\nu)}(E / \Lambda)}{\sqrt{J_{3}^{(\nu)}(E / \Lambda, 1)}} \\
\xi(w) & =\frac{J_{3}^{(\nu)}(E / \Lambda, w)}{J_{3}^{(\nu)}(E / \Lambda, 1)}
\end{aligned}
$$

Now we discuss the dependence of physical observables on parameters $E$ and $\nu$. Note that hereafter $E$ is given in units of $\Lambda(=460 \mathrm{MeV})$, At this point, it may be useful to stress that unlike in Ref. [8] there is no imaginary part in our matrix elements for any 
value of E since there is no $Q \bar{q}$ production threshold due to confinement. The value of $\mathrm{E}$ is not arbitrary, however. The first restriction comes from the requirement that the integral $J_{3}^{\nu}(E, 1)$ in Eq.(30) should be a positive definite quantity. Since this integral for $\nu>0$ is defined in terms of the derivatives of the confinement functions, it develops zeros above certain values of $\mathrm{E}$. The behavior of $J_{3}^{\nu}(E, 1)$ for $\nu=0,0.5,1$ is shown in Fig.1. Another restriction on E comes from the value of the heavy meson weak decay constants. From eq.(33) it is clear that our result in the heavy quark limit obeys the scaling relation $f_{B} \sqrt{m_{B}}=f_{D} \sqrt{m_{D}}$. The dependence of the weak decay constant values on $\mathrm{E}$ is shown in Fig.2, and it is clear that this quantity is very sensitive to the change in E: strongly increasing function of E. This is in line with what was observed in QCD sum rule approaches, see for example, [13]. In the present result the $\mathrm{E}=0$ case is unrealistically small, suggesting that even in the heavy quark limit E must be kept finite as it should be.

The values of the weak decay constants obtained in different models are in variance but eventually $f_{D}$ never exceeds $\sim 250 \mathrm{MeV}$ [13], [27], [28], [29], [30] when the pion decay constant is normalized to $f_{\pi}=132 \mathrm{MeV}$. We will use the above observations as a guide to constrain E. The numerical results for the upper bound obtained in this way for various choice of the infrared parameter $\nu$ are shown in Fig.3. It is interesting to note the lower bound for $\mathrm{E}$ obtained in Ref. [31] $\mathrm{E} \geq 0.5$, which might eventually be implemented to get the restriction on the infrared parameter $\nu$ which turns out as $\nu \leq 0.75$. Note, however, that there is an antithesis to the way this lower bound has been derived [32.

The IWF is plotted in Fig.4 for various values of $\mathrm{E}$ and $\nu$. One can see that the Edependence of this function is much weaker than that seen in the weak decay constants. This agrees qualitatively with the result obtained by using QCD sum rules [13], [14, [15]. Note, in particular, that in [13] the IWF is completely independent of E, which is rather curiuous intuitively. In the present result the E-dependence decreases for increasing $\nu$ : for $\nu=0$ and at maximum recoil the difference between the values of the IWF for $\mathrm{E}=0$ and $\mathrm{E}=1.0$ is no greater than $12 \%$. The general tendency is that the IWF is suppressed for larger values of $\mathrm{E}$.

On the other hand, the dependence on $\nu$ is much stronger as seen in Fig.5. For examlpe, at maximum recoil the IWF is suppressed by $13 \%$ for $\nu=0.5$, and by $23 \%$ for $\nu=1$ in comparison with $\nu=0$ when $\mathrm{E}=0$. A similar tendency is observed for other 
values of E.

The E-dependence of the slope parameter at zero recoil: $\rho^{2}=-\xi^{\prime}(1)$, which is an important quantity characterizing the IWF, is shown in Fig.6. One can see that it is contained within the following region:

$$
\begin{aligned}
& 0.42 \leq \rho^{2} \leq 0.64 \quad(\nu=0), \\
& 0.64 \leq \rho^{2} \leq 0.82 \quad(\nu=0.5), \\
& 0.62 \leq \rho^{2} \leq 0.64 \quad(\nu=1) .
\end{aligned}
$$

The result is consistent with the generous Bjorken lower bound: $\rho^{2} \geq 0.25$ [33: but somewhat less than the values obtained in other approaches (see, Table 2). It should be noted, however, that a recent fit to the CLEO $B \rightarrow D^{*} l \nu$ data with various analytic forms adopted for the IWF give $\rho^{2} \approx 1.1 \pm 0.5 \pm 0.3$, consistent with our present result [34] with $\nu \approx 0.5$. Also note that this quantity does not necessarily determine the global behavior of the IWF, as seen in Fig.8. Also, its accurate determination from experimental data is not trivial, in good part, due to the small count near zero recoil. The effort is being made from the CLEO data for the $B \rightarrow D^{*} l \bar{\nu}$ and we await a quick release of the analysis.

Plot of the IWF for the set of parameters $\{\nu, E\}$ providing the reasonable values for the weak decay constants (see, Table 1.) is presented in Fig.7. One can see that, for example, the curve with the parameters $\nu=0.75, \mathrm{E}=0.5$ (or $\mathrm{E}=230 \mathrm{MeV}$ ) is consistent with the experimental result within $19 \%$ off the best fit taken from Ref. [38]. In Fig.8 the IWF taken from the other approaches are shown.

\section{The effects of finite masses in the weak decays}

To study the correction to the heavy quark limit, we will take into account the effect of finite heavy quark masses in the weak decays of B and D mesons. This is of special interest since the QCD sum rule [13], [27] and the lattice calculations [28], [29] give an indication of a strong breakdown of the scaling law: 


$$
\begin{array}{cl}
f_{D}=170 \pm 30 \mathrm{MeV} & f_{B}=190 \pm 50 \mathrm{MeV} \\
f_{D}=150 \rightarrow 195 \mathrm{MeV} & f_{B}=150 \rightarrow 185 \mathrm{MeV} \\
f_{D}=200 \pm 25 \mathrm{MeV} & f_{B}=230 \pm 10 \mathrm{MeV} \\
f_{D}=185_{-3-7}^{+4+42} \mathrm{MeV} & f_{B}=160_{-6-19}^{+6+53} \mathrm{MeV} \\
f_{D}=208(9) \pm 35 \pm 12 \mathrm{MeV} & f_{B}=187(10) \pm 34 \pm 15 \mathrm{MeV}
\end{array}
$$

In 27] the spread in the value of the decay constants originates from varying the values of the heavy quark masses. Also the results are found without adopting any HQET, and the sum rule result with the method of Hilbert moments not listed here has given somewaht different values. Note that in [13] and 28] the complete break down of the scaling law even brings the value of $f_{D}$ lower than that of $f_{B}$.

To simplified the calculations, we consider only the case with $\nu=0$ in view of its very mild influence on the decay constants discussed earlier. Then we have

$$
f_{H} \sqrt{m_{H}}=\Lambda^{3 / 2} \frac{\sqrt{3}}{2 \pi} \frac{J_{2}^{(0)}\left(M_{Q} / \Lambda, E / \Lambda\right)}{\sqrt{J_{3}^{(0)}\left(M_{Q} / \Lambda, E / \Lambda\right)}}
$$

with the structural integrals defined as

$$
\begin{aligned}
& J_{2}^{(0)}(M, E)=4 \int_{0}^{\infty} \frac{d t t}{(1+t / M)^{3}}\left[t\left(1+\frac{t}{2 M}\right)-E\left(1+\frac{E}{2 M}\right)\right]\left\{a(z)+\frac{1}{2} \frac{t}{1+t / M} b(z)\right\} \\
& J_{3}^{(0)}(M, E)=4 \int_{0}^{\infty} \frac{d t t}{(1+t / M)^{3}}\left\{a(z)+\frac{t}{2}\left[3+\frac{t}{M}-\frac{(1+E / M)^{2}}{1+t / M}\right] b(z)\right\},
\end{aligned}
$$

where

$$
z=\frac{t}{1+t / M}\left\{t-2 E\left(1+\frac{E}{2 M}\right)\right\} .
$$


It is easy to show that eq.(33), whose structural integrals being defined in the Appendix, is reproduced in the heavy quark limit $M_{Q} \rightarrow \infty$.

We have adopted the values of the heavy mesons: $m_{D}=1.87 \mathrm{GeV}, m_{B}=5.27 \mathrm{GeV}$, and varied $E=m_{H}-M_{Q}$ which, in turn, determines the finite heavy quark masses. Just like in the heavy quark limit as discussed earlier, $f_{H}$ has turned out quite small for small $\mathrm{E}$, and the effect of the finite $M_{Q}$ is found rather insignificant. However, for $E \geq 0.5 f_{H}$ increases significantly. In order to compared with the result in the heavy quark limit as listed in Table 1, we we present here the finite quark mass result for $\mathrm{E}=1$ :

$$
f_{D}=175 \mathrm{MeV} \quad f_{B}=125 \mathrm{MeV}
$$

which is quite reasonable as compared with the lattice and QCD sum rule results quated above except that the B-meson decay constant is somewhat on the smaller side. When compared with our value in the heavy quark limit, the reduction is about $10 \%$ for the $\mathrm{B}$ meson and as large as $25 \%$ for the D meson. So obviously this effect is important and breaks the scaling law. The percentage reduction here is in agreement with what was found in ref.[13] within the QCD sum rule when the extra reduction factor $\left(M_{Q} / m_{H}\right)^{1.5}$ in this work is left out. We should note that it is this extra factor in this reference that eventually reverses the magnitudes of $f_{D}$ and $f_{B}$ (note that there the adopted value of $\mathrm{E}$ is somewhat smaller for $\mathrm{D}$ ), thus consistent with the lattice result in 28. In our present approach this reduction factor is apparently absent: we wonder if it is a model independent factor we have missed, or it is due to the specific way the parameters were defined in performing the Laplace sum rule in the heavy quark limit in [13]. In any event, we should stress that the present section is not intended as predicting the values of the heavy meson decay constants: rather it is to demonstrate the importance of the finite heavy quark mass effect to correct the heavy quark limit as long as this specific physical quantity is concerned, particularly for the D meson.

As for the finite quark mass effect on the IWF, we already studied it in our previous work [7] where $E \equiv 0$. The effect was found to be rather small and we expect a similar conclusion to hold here, thus has not been investigated. 


\section{Conclusion}

As an extension of our previous work [7], we have presented a quark model approach to the decay of heavy quark mesons where a confined light quark and the near on-shell infrared behavior of the heavy quarkpropagation are taken into account. The infrared behavior of the heavy quark is modelled in terms of a single parameter $\nu$ which modifies the simple free Feynman propagator. The study has been carried out in the heavy quark limit: $M_{Q} \rightarrow \infty$. However, we have retained the finite value for $E=m_{H}-M_{Q}$ : the difference between the heavy meson and heavy quark masses. We have found that the weak decay constant of the heavy quark mesons depends strongly on $\mathrm{E}$ while its $\nu$-dependence is rather mild. This fact has then been used to constrain the value of $\mathrm{E}$ by imposing a conservative upper bound for $f_{H}$ to be $\approx 250 \mathrm{MeV}$ for the $\mathrm{D}$ meson decay. In this way it is found that for a reasonable value $E \sim 460 \mathrm{MeV}$ with $\nu=0$ ( corresponding to a free heavy quark propagation) the value $f_{D}$ is found to be consistent with approaches like QCD sum rule [13], [27] and a lattice simulation [28], [29], [30].

In the same context we have studied the universal semi-leptonic decay form factor: the Isgur-Wise function. Unlike in the case of the decay constants, it is found to be controlled more dominantly by the infrared parameter $\nu$ which gives a desirable suppression of this function for $\nu>0$. As an example, for a set of parameters: $\mathrm{E}=0$ and $\nu=0.75$ giving reasonable values for the weak decay constants, the IWF turns out to be consistent with the best empirical fit to the ARGUS data [37] nearly within the experimental error bars: we may need a little extra suppresion.

Our treatment of the infrared behavior of the heavy quark propagator in terms of a single parameter may seem too simple. It is motivated by studies in the abelian gauge field, thus is not rooted from real QCD. However, we believe that it certainly points to the direction of further investigation, as it appears reasonable enough to believe that a free heavy quark propagator (with a simple pole at the quark mass) may not be realistic but needs to be modified in such a way to respect the heavy quark symmetry.

We have also studied the effect of the finite heavy quark mass, and found that it gives a significant deviation from the scaling law for the weak decay constants obtained in the heavy quark limit (and also in the non-relativistic quark models). We thus confirmed the claims from certain lattice and QCD sum rule approaches. 
To conclude, we want to reiterate the following: while the importance of retaining the finite value for $\mathrm{E}$ appears to be established in other approaches, the possible significance of the infrared behavior of the heavy quark propagator has not been paid much attention except in a simple model [9]. While we ourselves are in persuit of a more realistic form of the heavy quark infrared propagator, we will certainly feel happy if the present work may invoke certain interest in this direction.

\section{ACKNOWLEDGMENTS}

We would like to thank N.Isgur, A.Radyushkin, J. Körner, T. Mannel and M. Neubert

for useful discussions. This work was supported in part by the United States Department of Energy under Grant No. DE-FG05-84-ER40413. 


\section{APPENDIX}

\section{Two-point function.}

$$
\begin{aligned}
J_{2}^{(\nu)}(E) & =\int \frac{d^{4} k}{\pi^{2} i} \int d \sigma_{z} \frac{z-k v}{\left(z^{2}-k^{2}\right)(-E-k v)^{1+\nu}} \\
& =\frac{\Gamma(2+\nu)}{\Gamma(1+\nu)} \int_{0}^{1} d \alpha(1-\alpha)^{\nu} \int \frac{d^{4} k}{\pi^{2} i} \int d \sigma_{z} \frac{z-k v}{\left[\alpha\left(z^{2}-k^{2}\right)-(1-\alpha)(E+k v)\right]^{2+\nu}} \\
& =\frac{\Gamma(2+\nu)}{\Gamma(1+\nu)} \int_{0}^{\infty} d t t^{\nu} \int_{0}^{\infty} d u u \int d \sigma_{z} \frac{z+t / 2}{\left[z^{2}+u-t E+t^{2} / 4\right]^{2+\nu}}
\end{aligned}
$$

(1) $0 \leq \nu<1$

$$
J_{2}^{(\nu)}(E)=2^{\nu} \frac{\sin \pi \nu}{\pi \nu} \int_{0}^{\infty} d u u^{(1-\nu) / 2} \int_{0}^{1} d x \frac{x^{(\nu-1) / 2}}{(1-x)^{\nu}}\{a(u-2 E \sqrt{x u})+\sqrt{x u} b(u-2 E \sqrt{x u})\} .
$$

(2) $\nu=1$

$$
J_{2}^{(\nu)}(E)=2 \int_{0}^{\infty} d u\{a(u-2 E \sqrt{u})+\sqrt{u} b(u-2 E \sqrt{u})\} .
$$

\section{Three-point function.}

$$
\begin{aligned}
J_{3}^{(\nu)}(E, w) & =\int \frac{d^{4} k}{\pi^{2} i} \int d \sigma_{z} \frac{z-\left(k v+k v^{\prime}\right) /(w+1)}{\left(z^{2}-k^{2}\right)(-E-k v)^{1+\nu}\left(-E-k v^{\prime}\right)^{1+\nu}} \\
& =\frac{\Gamma(2+2 \nu)}{\Gamma^{2}(1+\nu)} \int_{0}^{1} d \beta \beta^{\nu}(1-\beta)^{\nu} \int \frac{d^{4} k}{\pi^{2} i} \int d \sigma_{z} \frac{z-\left(k v+k v^{\prime}\right) /(w+1)}{\left(z^{2}-k^{2}\right)\left[-E-k\left(\beta v+(1-\beta) v^{\prime}\right)\right]^{2+2 \nu}} \\
& =2^{1+2 \nu} \frac{\Gamma(1+2 \nu)}{\Gamma^{2}(1+\nu)} \int_{0}^{1} d \tau \frac{\tau^{\nu}(1-\tau)^{\nu}}{W^{1+\nu}} \int_{0}^{\infty} d u u^{\nu} \int d \sigma_{z} \frac{z+\sqrt{u / W}}{\left[z^{2}+u-2 E \sqrt{u / W}\right]^{1+2 \nu}}
\end{aligned}
$$

with $\mathrm{W}$ given by 


$$
W=1+2 \tau(1-\tau)(w-1)
$$

(1) $\nu=0$

$$
J_{3}^{(\nu)}(E)=2 \int_{0}^{1} \frac{d \tau}{W} \int_{0}^{\infty} d u\{a(u-2 E \sqrt{u / W})+\sqrt{u / W} b(u-2 E \sqrt{u / W})\} .
$$

(2) $\nu=0.5$

$$
\begin{aligned}
& J_{3}^{(\nu)}(E)=\frac{16}{\pi} \int_{0}^{1} d \tau \frac{\sqrt{\tau(1-\tau)}}{W^{3 / 2}} \int_{0}^{\infty} d u \sqrt{u}\left\{a_{1}(u-2 E \sqrt{u / W})+\sqrt{u / W} b_{1}(u-2 E \sqrt{u / W})\right\} . \\
& (3) \nu=1 \\
& J_{3}^{(\nu)}(E)=8 \int_{0}^{1} d \tau \frac{\tau(1-\tau)}{W^{2}} \int_{0}^{\infty} d u u\left\{a_{2}(u-2 E \sqrt{u / W})+\sqrt{u / W} b_{2}(u-2 E \sqrt{u / W})\right\},
\end{aligned}
$$

where $f_{i}(u)=(-)^{i} f^{(i)}(u),(f=a, b)$.

(4) $0<\nu<0.5$

$$
\begin{aligned}
J_{3}^{(\nu)}(E)= & 2^{1+2 \nu} \frac{\sin 2 \pi \nu}{2 \pi \nu} \frac{\Gamma(1+2 \nu)}{\Gamma^{2}(1+\nu)} \int_{0}^{1} d \tau \frac{\tau^{\nu}(1-\tau)^{\nu}}{W^{1+\nu}} \int_{0}^{1} d x \frac{x^{\nu}}{(1-x)^{2 \nu}} \int_{0}^{\infty} d u u^{1-\nu} \\
& \left\{a_{1}(u-2 E \sqrt{u x / W})+\sqrt{u x / W} b_{1}(u-2 E \sqrt{u x / W})\right\} .
\end{aligned}
$$

(4) $0.5<\nu<1$

$$
\begin{aligned}
J_{3}^{(\nu)}(E)= & \frac{2^{2 \nu}}{\nu} \frac{\sin \pi(2 \nu-1)}{\pi(2 \nu-1)} \frac{\Gamma(1+2 \nu)}{\Gamma^{2}(1+\nu)} \int_{0}^{1} d \tau \frac{\tau^{\nu}(1-\tau)^{\nu}}{W^{1+\nu}} \int_{0}^{1} d x \frac{x^{\nu}}{(1-x)^{2 \nu-1}} \int_{0}^{\infty} d u u^{2-\nu} \\
& \left\{a_{2}(u-2 E \sqrt{u x / W})+\sqrt{u x / W} b_{2}(u-2 E \sqrt{u x / W})\right\} .
\end{aligned}
$$




\section{References}

[1] N.Isgur and M.B.Wise, Phys. Lett. B232, 113 (1989); B237, 527 (1990).

[2] H.Georgi, Phys. Lett. B240, 447 (1990);

B. Grinstein, Nucl. Phys. B399, 253 (1990);

A. Falk, H. Georgi, and M. Wise, Nucl. Phys. B343, 1 (1990);

T. Mannel, W. Roberts, and Z. Ryzak, NUcl. Phys. B368, 204 (1992).

[3] A.F.Falk, B.Grinstein, and M.E.Luke, Nucl. Phys. B357, 185 (1991).

[4] N.Isgur, D.Scora, B.Grinstein, and M.B.Wise, Phys. Rev. D39, 799 (1989); Phys. Rev. D40, 1491 (1989).

[5] F. E. Close and A. Wambach, Nucl. Phys. B412, 167 (1994).

[6] M.Neubert and V.Rieckert, Nucl. Phys. B382, 97 (1992);

M.Neubert, Phys. Lett. B264, 455 (1991).

[7] M.A.Ivanov, O.E.Khomutenko, and T.Mizutani, Phys. Rev. D46, 3817 (1992).

[8] B.Holdom and M.Sutherland, Phys. Rev. D47, 5067 (1993).

[9] A.I.Karanikas and C.N.Ktorides, Phys. Lett. B301, 397 (1993).

[10] M.Sadzikowski and K.Zalewski, hep-ph/9304319, 1993.

[11] T.Kugo, M.G. Mitchard, and Y.Yoshida, hep-ph/9312267, 1993.

[12] A.V.Radyushkin, Phys. Lett. B271, 218 (1991).

[13] M. Neubert, Phys. Rev.D45, 2451 (1992).

[14] P. Ball, Phys. Lett. B281, 133 (1992).

[15] P. Colangelo, G. Nardulli, and N. Paver, Phys. Lett. B293, 207 (1992).

[16] C.W. Bernard et al. hep-lat/9307005, 9312038, 1993.

[17] UKQCD Collaboration, hep-ph/9312241, hep-lat/9308019, 9312088, 1993.

[18] M.A. Shifman, A.I. Vainshtein, and V.I. Zakharov,

Nucl. Phys. B147, 385; 448 (1979).

[19] E. de Rafael and J. Taron, Phys. Lett. 282, 215 (1992). 
[20] A.F.Falk, M.Luke, and M.B.Wise, Phys. Lett.B299, 123 (1993).

[21] B. Grinstein and P.F. Mende, Phys. Lett. B299, 127 (1993).

[22] C.E. Carloson, J. Milana, N. Isgur, T. Mannel, and W. Roberts, Phys. Lett. B299, 133 (1993).

[23] C.A.Dominguez, J.G.Köerner, and D.Pirjol Phys. Lett. B301, 257 (1993).

[24] E. de Rafael and J. Taron, hep-ph/9306214, 1993.

[25] G.V.Efimov and M.A.Ivanov, The Quark Confinement Model of Hadrons, (IOP Publishing, Bristol \& Philadelphia, 1993).

[26] B. M. Barbashov, Soviet Phys. JETP 21, 402 (1965).

[27] C.A. Dominguez and N. Paver, Phys. Lett. B197, 423 (1987);

C. A. Dominguez, SISSA Ref 139/93/EP, (to be published in Proc. 3rd Workshop on the Tau-Charm Factory, Marbella, Spain, June 1993).

[28] A. Abada, et al. Nucl. Phys. B376, 172 (1992).

[29] UKQCD Collaboration, Phys. Rev. D49, 1594 (1994).

[30] C.W. Bernard, J.N. Labrenz, A. Soni, Phys. Rev. D49, 2536 (1994).

[31] Z. Guralnik and A.V. Manohar, Phys. Lett. B302, 103 (1993).

[32] I. I. Bigi and N. G. Uraltsev, CERN-TH.7091/93 (hep-ph/9311337) (1993).

[33] J.D.Bjorken, Preprint SLAC-PUB-5278 (1990).

[34] D. Z. Besson, Proc. 16th Intl. Symposium on Lepton Photon Interactions, Cornell, p221 (1993).

[35] J.L.Rosner, Phys. Rev. D42, (1990) 3732.

[36] T.Mannel, W.Roberts, and Z.Ryzak, Phys.Lett. B254, 274 (1990).

[37] ARGUS Collaboration, Z.Phys. C57,533 (1993).

[38] S. Stone. in B decays, edited by S. Stone (World Scientific, Singapore, 1992), p.158. 


\section{List of Figures}

Fig.1. The dependence of the structural integral $J_{3}^{\nu}(E, 1)$ (in arbitrary units) on E for various choices of the infrared parameter $\nu$.

Fig.2. The dependence of the weak decay constants on $\mathrm{E}$ for various choices of the infrared parameter $\nu$.

Fig.3. The correlation of $\mathrm{E}$ and $\nu$ which provides the weak decay constants: $f_{D} \sim 250$ $\mathrm{MeV}$, and $f_{B} \sim 150 \mathrm{MeV}$.

Fig.4. The IW function for various choices of $\mathrm{E}$ with the fixed value of infrared parameter $\nu$.

Fig.5. The IW function for various choices of the infrared parameter $\nu$ with fixed E.

Fig.6. Slope parameter (ar zero recoil) as a function of $\mathrm{E}$ for various choices of the infrared parameter $\nu$.

Fig.7. Plot of the IW function for the set of parameters $\{\nu, E\}$ providing the reasonable values for the weak coupling constants (see, Table 1.). The data points are taken from Ref. [37]. The dash curve represents the best fit of $e^{-\rho^{2}(w-1)}$ with $\rho^{2}=1.18 \pm 0.50$ taken from Ref. [38]. The dot curves represent the upper and lower bounds of the fit that related to the experimental uncertainties.

Fig.8. Plot of the IW functions taken from the other approaches: VQM (valence quark model) [4]; QCD (QCD sum rules) [12]; ROM (relativistic quark model) [6]; INFRA (infrared heavy quark propagator) [9]; The data points are taken from Ref. 37]. FIT (the best fit of experimental data) [38]. 


\section{List of Tables}

Table 1. The magnitudes of weak decay constants and slope parameter of IW function for some set of parameters $\{\nu, E\}$.

Table 2. The slope parameter of IW function in other approaches including our previous work [7]. 
Table 1.

\begin{tabular}{|c|c|c|c|}
\hline \hline & $\begin{array}{c}\nu=0.00 \\
\mathrm{E}=460 \mathrm{MeV}\end{array}$ & $\begin{array}{c}\nu=0.50 \\
\mathrm{E}=276 \mathrm{MeV}\end{array}$ & $\begin{array}{c}\nu=0.75 \\
\mathrm{E}=230 \mathrm{MeV}\end{array}$ \\
\hline$f_{D}(\mathrm{MeV})$ & 231 & 216 & 226 \\
\hline$f_{B}(\mathrm{MeV})$ & 137 & 140 & 135 \\
\hline$\rho^{2}$ & 0.61 & 0.83 & 0.92 \\
\hline \hline
\end{tabular}

Table 2.

\begin{tabular}{|l|l|}
\hline \hline Bjorken [33] & $>\frac{1}{4}$ \\
\hline Isgur [4] & $0.63(0.33)$ \\
\hline Rosner [35] & $1.44 \pm 0.41$ \\
\hline Mannel [36] & $1.77 \pm 0.74$ \\
\hline Neubert [13] & $1.28 \pm 0.25$ \\
\hline Bernard [16] & $1.41 \pm 0.19 \pm 0.41$ \\
\hline UKQCD Collab. [17] & $1.2_{-8}^{+7}$ \\
\hline Radyushkin [12] & $\infty$ \\
\hline Karanikas [9] & 0 \\
\hline Sadzikowski [10] & 1.24 \\
\hline Kugo [1] & $1.8-2.0$ \\
\hline Ivanov et al. [7] & 0.43 \\
\hline \hline
\end{tabular}

